Pacific Journal of Mathematics

THE SPECTRAL DENSITY OF A STRONGLY MIXING 


\title{
THE SPECTRAL DENSITY OF A STRONGLY MIXING STATIONARY GAUSSIAN PROCESS
}

\author{
ERIC HAYASHI
}

\begin{abstract}
Let $w$ be a nonnegative integrable weight function on the real line $R$ such that $(\log w) /\left(1+x^{2}\right)$ is also integrable. Let $F_{T}$ and $P_{T}$ denote, respectively, the closed linear spans in $L^{2}(R, w d x)$ of $\left\{e^{i a x}: a \geqq T\right\}$ and $\left\{e^{i a x}: a \leqq T\right\}$. Let $\theta(T)$ denote the angle between $P_{0}$ and $F_{T}$. The problem considered here is that of describing those weights $w$ for which $\theta(T) \rightarrow \pi / 2$ as $T$ tends to infinity (such weights arise as the spectral densities of strongly mixing stationary Caussian processes). Some necessary conditions on $w$ are given for $\theta(T) \rightarrow \pi / 2$, and a construction is given to show that $w$ may have arbitrarily wild oscillatory discontinuities even if $\theta(T) \rightarrow \pi / 2$. Another measure of the interdependence of $P_{0}$ and $F_{T}$ is introduced: let $\theta *(T)$ denote the angle between $P_{T} \ominus\left(P_{T} \cap F_{0}\right)$ and $F_{0} \ominus\left(P_{T} \cap F_{0}\right)$. A complete structural char acterization is given of those weights $w$ for which both $\theta(T)$ and $\theta *(T)$ tend to $\pi / 2$. Moreover, it is shown that if either $\theta(T)$ or $\theta *(T)$ is eventually positive and the other tends to $\pi / 2$, then they both do.
\end{abstract}

Let $W$ denote the class of weights $w$ for which $\theta(T)$ tends to $\pi / 2$. These weights arise as the spectral densities for stationary Gaussian processes which satisfy a certain strong mixing condition. Helson and Sarason studied the analogous class of weights on the unit circle, which correspond to discrete-time processes. In [10] and [17] they give a structual characterization of these weights as those of the from $|P|^{2} e^{u+\tilde{v}}$, where $P$ is a polynomial and where $u$ and $v$ are continuous functions on the unit circle ( $\widetilde{v}$ denotes a harmonic conjugate of $v$ ). Since the entire functions of exponential type are related to the bounded uniformly continuous functions on $R$ rougly in the same way as polynomials are related to continuous functions on the unit circle, it is tempting to conjecture that $W$ is precisely the class $W_{1}$ of weights $w$ which have the form $w=|B|^{2} e^{u+\tilde{\nu}}$, where $B$ is an entire function of exponential type and is square-summable on $R$, and where $u$ and $v$ are in $B U C$, the space of bounded uniformly continuous functions on $R$. The class $W_{1}$ is discussed in $\S 4$. It is easy to show that $W_{1} \subset W$, but whether or not $W \subset W_{1}$ remains unanswered. A necessary and sufficient condition for a weight function in $W$ to be in $W_{1}$ is given in Theorem 2 of that section; it is hoped that further investigation of that condition will lead to an answer. Section 6 contains some partial results in this direction. 
The class $W_{*}$ of weights for which $\theta^{*}(T) \rightarrow \pi / 2$ is taken up in $\S 5$.

2. Some preliminaries. Let $L^{\infty}$ denote the space of essentially bounded measurable functions on the real line, and let $H^{\infty}$ denote the space of functions in $L^{\infty}$ whose Poisson extensions into the upper half-plane are analytic. This section will outline some facts about the closed algebras between $H^{\infty}$ and $L^{\infty}$ which will be needed either in substance or for motivation in the following sections.

Let $A$ be a closed subalgebra of $L^{\infty}$ which contains $H^{\infty}$. It was shown by Marshall and Chang in [16] and [4] that $A$ is generated by $H^{\infty}$ and the complex conjugates of the inner functions which are invertible in $A$; such algebras have been named Douglas algebras. The main algebra we will consider is $H^{\infty}\left[e^{-i x}\right]$, the closed algebra generated by $H^{\infty}$ and the function $e^{-i x}$. In [19], Sarason showed that this algebra equals the algebraic sum $H^{\infty}+B U C$, where $B U C$ denotes the space of bounded uniformly continuous function on $\boldsymbol{R}$; $B U C$ is also the $C^{*}$-algebra generated by the inner functions which are invertible in $H^{\infty}+B U C$. It turns out that this form holds for all Douglas algebras. In [3], Chang proved the first two parts of the following theorem which will be used in proving Theorem 5 . Part (iii) was proved by Chang and Marshall in [4].

THEOREM. Let $A$ be a closed subalgebra of $L^{\infty}$ which contains $H^{\infty}$, and let $C_{A}$ denote the $C^{*}$-algebra generated by the inner functions which are invertible in $A$. Then the following statements are true:

(i ) $A=H^{\infty}+C_{A}$

(ii) $A \cap \bar{A}=L^{\infty} \cap\left(C_{A}+\widetilde{C}_{A}\right)$ where $\bar{A}$ denotes the space of complex conjugates of functions in $A$, and $\widetilde{C}_{A}$ denotes the space of harmonic conjugates of functions in $C_{A}$.

(iii) If $f$ is a function in $C_{A}$, then $\operatorname{dist}\left(f, H^{\infty}\right)=\operatorname{dist}\left(f, H^{\infty} \cap C_{A}\right)$, where $\operatorname{dist}(f, S)=\inf \left\{\|f-g\|_{\infty}: g \in S\right\}$.

The role of the conjugation operator in the study of Douglas algebras is tied largely to results discovered by Fefferman and Stein in [9]. If $f$ is a locally integrable function and $I$ a finite interval, we let $f_{I}=|I|^{-1} \int_{I} f(x) d x$, where $|I|$ denotes the length of $I$. A function is said to be of bounded mean oscillation, or to lie in $B M O$, if the quantity $\|f\|_{*}=\sup _{I}|I|^{-1} \int_{I}\left|f(x)-f_{I}\right| d x$ is finite (the supremum being taken over all finite intervals). If functions in $B M O$ which differ by a constant are identified, then $B M O$ becomes a Banach space, with norm \|\|$_{*}$, which Fefferman and Stein identified in the above paper as the dual of $H^{1}$. They also showed that the 
functions $f$ in $B M O$ are precisely those of the form $f=u+\widetilde{v}$ where $u$ and $v$ are in $L^{\infty}$, and that the conjugation operator is a bounded map from $L^{\infty}$ into $B M O$, which is a fact we will need later.

Another important class of functions is $V M O$, the functions of vanishing mean oscillation. A function $f$ in $B M O$ is said to be in $V M O$ if the numbers $M_{a}(f)=\sup _{|I| \leqq a}|I|^{-1} \int_{I}\left|f(x)-f_{I}\right| d x$ tend to zero as $a$ tends to zero. It is easy to see that a uniformly continuous function in $B M O$ belongs to $V M O$. It is also well known that the conjugation operator preserves certain smoothness properties of functions (see [5]), so it is not surprising that if $u$ and $v$ are uniformly continuous functions in $B M O$, then $u+\widetilde{v}$ is in VMO. In [19], Sarason proved that the converse is also true. He also showed that $V M O$ plays an important role in the structure of $H^{\infty}+B U C$, namely that $\left(H^{\infty}+B U C\right) \cap \overline{H^{\infty}+B U C}=L^{\infty} \cap V M O$. In [3], Chang associated to each Douglas algebra $A$ a space $V M O_{A}$ which is a generalized version of $V M O$. (Roughly speaking, functions in $V M O_{A}$ look locally like $V M O$ functions where the functions in $C_{A}$ are nicly behaved.) Furthermore, it is shown there that $V M O_{A} \cap L^{\infty}=A \cap \bar{A}$ and $V M O_{A}=C_{A}+\widetilde{C}_{A}$.

3. The class $W$. We shall only be considering integrable weights $w$ which satisfy the condition that $(\log w) /\left(1+x^{2}\right)$ is integrable, so $w$ can be expressed in the form $w=|h|^{2}$ where $h$ is an outer function in $H^{2}$, the usual Hardy space for the upper half-plane (see [5, p. 83]). For each nonnegative real number $T$, define the number

$$
\rho(w, T)=\sup _{f_{1} f_{2}}\left|\int_{-\infty}^{\infty} e^{i T x} f_{1}(x) \overline{f_{2}}(x) w(x) d x\right|,
$$

where $f_{1}$ and $f_{2}$ are allowed to run over the unit balls of $F_{0}(w)$ and $P_{0}(w)$, respectively. The number $\rho(w, T)$ is just the cosine of the angle $\theta(T)$ between the subspaces $P_{0}(w)$ and $F_{T}(w)$ of $L^{2}(\boldsymbol{R}, w d x)$. Let $W$ denote the class of all integrable weights $w$ for which $\rho(x, T) \rightarrow 0$ as $T \rightarrow \infty$.

The following lemma is a variation on a theme by Helson and Szegö [11].

Lemma 1. Let $w=|h|^{2}$ where $h$ is outer in $H^{2}$. Then $w$ is in $W$ if and only if the function $\bar{h} / h$ belongs to the algebra $H^{\infty}+B U C$.

Proof. The idea here is that $\rho(w, T)$ equals $\operatorname{dist}\left(e^{i T x} \bar{h} / h, H^{\infty}\right)$, the distance in $L^{\infty}$ of the function $e^{i T x} \bar{h} / h$ to the space $H^{\infty}$. To see this, note that the unit ball of $F_{0}(w)$ is the closure in $L^{2}(\boldsymbol{R}, w d x)$ of 
functions $g$ in $H^{2}$ which satisfy $\int|g h|^{2}=\int|g|^{2} w \leqq 1$. Thus, we have

$$
\begin{aligned}
\rho(w, T) & =\sup _{g_{1} g_{2}}\left|\int_{-\infty}^{\infty} e^{i T x}\left(g_{1} h\right)\left(g_{2} h\right) \cdot \frac{\bar{h} \cdot h}{h \cdot h} d x\right| \\
& =\sup _{f_{1} f_{2}}\left|\int_{-\infty}^{\infty} e^{i T x} f_{1} f_{2} \frac{\bar{h}}{h} d x\right|,
\end{aligned}
$$

where $f_{1}$ and $f_{2}$ are the functions $g_{1} h$ and $g_{2} h$, respectively. Since $h$ is outer, the $f_{i}$ range independently over a dense subset of the unit ball of $H^{2}$, hence their product ranges over a dense subset of the unit ball of $H^{1}$. Now $H^{\infty}$ is the annihilator in $L^{\infty}$ of $H^{1}$, so $\rho(w, T)$ equals the norm of $e^{i T x} \bar{h} / h$ in $L^{\infty} / H^{\infty}$, which equals $\inf _{f \in H^{\infty}}\left\|e^{i T x}(\bar{h} / h)-f\right\|_{\infty}$. Thus $w$ is in $W$ if and only if $\operatorname{dist}\left(e^{i T x} \bar{h} / h, H^{\infty}\right) \rightarrow 0$ as $T \rightarrow \infty$, end Lemma 1 is proved.

The following theorem is stated without proof in [13]. The proof given here is essentially the same as the Helson-Sarason proof in [11]; the argument is sketched below since it will be used on several occasions.

THEOREM 1. Let $w=|h|^{2}$ where $h$ is outer in $H^{2}$. Then $w$ is in $W$ if and only if, for every $\varepsilon>0, w$ can be written in the form $w=\left(1+x^{2}\right)\left|B_{\varepsilon}\right|^{2} e^{u_{\varepsilon}+v_{\varepsilon}}$ where $u_{\varepsilon}$ and $v_{\varepsilon}$ are real functions on $R$ with $\left\|u_{\varepsilon}\right\|_{\infty}+\left\|v_{\varepsilon}\right\|_{\infty}<\varepsilon$ and where $B_{\varepsilon}$ is an entire function of exponential type which is bounded on the real axis and zero free in the upper half-plane.

Proof. First suppose that $w=\left(1+x^{2}\right)|B|^{2} e^{u+\tilde{v}}$ where $\|u\|_{\infty}+$ $\|v\|_{\infty}<\varepsilon$ and $B$ is entire of exponential type and bounded on $R$ with no zeros in the upper half-plane. Since $(\log |B|)\rangle\left(1+x^{2}\right)$ is integrable on $R$, it follows from Nevanlinn's representation theorem ([8, p. 22]) that $e^{i T x} B$ is an outer function for some $T$, so

$$
h=(x+i) e^{i T x} B e[(u+\widetilde{v})+i(\widetilde{u}-v)] / 2,
$$

and

$$
\bar{h} / h=\frac{x-i}{x+i} e^{-2 i T x}\left[B^{*}(x) / B(x)\right] e^{i(v-\tilde{u})}
$$

where $B^{*}(z)=\overline{B(\bar{z})}$. Furthermore, by Nevanlinna's theorem, the zeros of $B^{*}$ form a Blaschke sequence for the upper half-plane, so $B^{*} / B=b \cdot e^{i \tau x}$ for some Blaschke product $b$ and real number $\tau$. The factor $e^{i \tau x}$ may be absorbed by the factor $\bar{e}^{2 i T x}$, so 


$$
\begin{aligned}
\operatorname{dist}\left(e^{2 i T x} \bar{h} / h, H^{\infty}\right) & =\operatorname{dist}\left(\frac{x-i}{x+i} b e^{i(v-\tilde{u})}, H^{\infty}\right) \\
& =\operatorname{dist}\left(e^{i(v-\tilde{u})}, \bar{b} \frac{x-i}{x+i} H^{\infty}\right) \\
& \leqq \operatorname{dist}\left(e^{i(v-\tilde{u})}, H^{\infty}\right) .
\end{aligned}
$$

To get an estimate on this last quantity, let $g=e^{-(u+i \tilde{u})}$. Then $g$ is in $H^{\infty}$, and

$$
\begin{aligned}
\left\|e^{\imath(v-\tilde{u})}-g\right\|_{\infty} & =\left\|1-g \cdot e^{-i(v-\tilde{u})}\right\|_{\infty} \\
& =\left\|1-e^{-u-i v}\right\|_{\infty} \\
& \leqq\left[\left[\varepsilon \cdot\left(e^{\varepsilon}\right)\right]^{2}+\left(e^{\varepsilon}-1\right)^{2}\right]^{1 / 2} .
\end{aligned}
$$

Since this last expression tends to zero as $\varepsilon \rightarrow 0$, and $\rho(w, T)$ is a nonincreasing function of $T$, it follows that $\lim _{T \rightarrow \infty} \rho(w, T)=0$.

Suppose now that $w$ is a weight in $W$. Then, if $\varepsilon>0$ is given, for some positive $T$, there exists a function $A$ in $H^{\infty}$ such that $\bar{h} / h=e^{-i T x} A \cdot e^{s+i t}$ where $s$ and $t$ are real functions with $\|s\|_{\infty}+\|t\|_{\infty}<$ $\varepsilon / 2$. Thus the inequality

$$
0<|h(x)|^{2} e^{-s-\tilde{t}}=e^{-i T x} A(x) h^{2}(x) e^{-\tilde{t}(x)+i t(x)}
$$

holds almost everywhere on $R$. There is no harm in assuming that $\varepsilon<\pi / 2$. This insures that $\operatorname{Re}\left[\bar{e}^{\tilde{t}+i t}\right] \geqq 0$ so that the last factor on the right is actually the boundary function for a function in $(z+i)^{2} \cdot H^{1}$ (see [5, p. 34]). So

$$
S(\boldsymbol{z})=e^{-\imath T z} A(\boldsymbol{z}) h^{2}(\boldsymbol{z}) e^{-\tilde{t}(z)+i t(z)}
$$

is a function which is analytic in the upper half-plane, positive a.e. on the real axis, and is in $H^{1 / 2}$ of every half-disk with diameter on the real axis. Using a fact about analytic continuation noted by Helson and Sarason (seen [14]), we can analytically continue $S$ across the real axis by reflection across the diameters of arbitrarily large half-disks. Thus $S$ is an entire function. That it is of exponential type follows from a theorem of Krein which says that an entire function which is of bounded characteristic in both upper and lower half-planes is of exponential type. However, the following direct estimate obtained by Koosis in [14] is more useful:

$$
|S(z)| \leqq C \cdot\left(1+|z|^{2}\right) e^{\mid T^{\operatorname{Im} z \mid}},
$$

where $C$ is a constant independent of $z$. Now, since $(\log S) /\left(1+x^{2}\right)$ is summable, $S$ can be factored as $S(x)=B_{1}^{*}(x) B_{1}(x)$ on the real axis, where $B_{1}$ is entire of exponential type at most $T / 2$ and has no zeros in the upper half-plane (see [2, p. 125]). If $B_{1}$ has no zeros, 
then $B_{1}(x)=e^{a+c x}$ for some constants $a$ and $c$. But the constant $c$ must be purely imaginary for $\left(\log \left|B_{1}\right|\right) /\left(1+x^{2}\right)$ to be integrable, and in this case, we can assume that $B_{1}$ is constant. So, without loss of generality, $B_{1}$ has a root $z_{0}$. Let $B_{2}=B_{1} /\left(z-z_{0}\right)$. Then for real $x$, we have $\left|B_{2}(x)\right|^{2}=\left|S(x) /\left(x-z_{0}\right)\right|^{2}$ which is bounded on $\boldsymbol{R}$ by the Koosis estimate. Furthermore,

$$
w=|h|^{2}=S(x) e^{s+\tilde{t}}=\left(1+x^{2}\right) \cdot\left|B_{2}(x)\right|^{2} e^{r+s+\tilde{t}}
$$

where $r=\log \left|x-z_{0} / x-i\right|^{2}$. Since the function $e^{r}$ is in $B U C$, it can be uniformly approximated by entire functions of exponential type (see [2, p. 249]). Thus, we can write $e^{r}=\left|B_{3}\right|^{2} e^{s_{1}}$ where $B_{3}$ is entire of exponential type and $\left\|s_{1}\right\|_{\infty}<\varepsilon / 2$. Putting all this together yields

$$
w=\left(1+x^{2}\right) \cdot B_{2} B_{2}^{*} B_{3} B_{3}^{*} e^{s_{1}+s+\tilde{t}} .
$$

Now $B_{2} B_{2}^{*} B_{3} B_{3}^{*}$ can be factored as $B B^{*}$ on the $x$-axis where $B$ is an entire function of the desired type. Setting $u_{\varepsilon}=s_{1}+s$ and $v_{\varepsilon}=t$ gives the desired result.

The following corollary gives local versions of properties stated in [10] for weights $w$ in $W$. Corollary 2 will be used in proving Theorem 3.

CoRollary 1. If $w$ is in $W$, there is a unique sequence $\left(r_{n}\right)$ of real numbers such that, if $B_{0}$ is the Hadamard product with zeros $\left(r_{n}\right)$, then the following are true:

(1) $w=\left|B_{0}\right|^{2} e^{f}$ where $f$ is a function which is of vanishing mean oscillation on every finite interval. ly for every finite interval $I^{\circ}$

(2) $\lim _{|J| \rightarrow 0, J \subset I} \sup |J|^{-2}\left[\int_{J}\left(w /\left|B_{0}\right|^{2}\right) d x\right]\left[\int_{J}\left(\left|B_{0}\right|^{2} / w\right) d x\right]=1$ uniform-

(3) $w /\left|B_{0}\right|^{2}$ has an antiderivative which is uniformly smooth on every finite interval $I$, i.e.,

$$
w_{I}(a)=\left.\sup _{x \in I,|h| \leqq a}\left|\int_{x}^{x+h} w /\right| B_{0}\right|^{2}-\int_{x-h}^{x} w /\left|B_{0}\right|^{2}|/| \int_{x-h}^{x+h} w /\left|B_{0}\right|^{2} \mid
$$

tends to zero uniformly as a tends to zero for each finite interval I.

(4) $w$ cannot have a jump discontinuity.

Proof. Suppose that $w=\left(1+x^{2}\right)\left|B_{1}\right|^{2} e^{u_{1}+\tilde{v}_{1}}$ and $w=\left(1+x^{2}\right)\left|B_{2}\right|^{2} e^{u_{2}+\tilde{v}_{2}}$ are two representations of $w$ given by Theorem 1 . Then $\left|B_{1} / B_{2}\right|^{2}$ and $\left|B_{2} / B_{1}\right|^{2}$ are both locally summable, provided that $\left\|v_{1}-v_{2}\right\|_{\infty}<\pi / 2$. Thus, if $v_{1}$ and $v_{2}$ are small enough, $B_{1}$ and $B_{2}$ must have the same real zeros, counting multiplicity. Let $B_{0}$ denote the Hadamard product with these zeros. Then, for every small positive $\varepsilon$, you can 
write $f=\log \left(w /\left|B_{0}\right|^{2}\right)=\log \left|B_{\varepsilon} / B_{0}\right|^{2}+\log \left(1+x^{2}\right)+u_{\varepsilon}+\widetilde{v}_{\varepsilon} \quad$ where $\left\|u_{\varepsilon}\right\|_{\infty}+\left\|v_{\varepsilon}\right\|_{\infty}<\varepsilon$. Now, the first two terms on the right are continuous; let $g$ denote their sum. Then it is clear that, for any finite interval $I$,

$$
\begin{aligned}
\lim _{\substack{|J| \rightarrow 0 \\
J \subset I}} \sup |J|^{-1} \int_{J}\left|f-f_{J}\right| & \leqq \lim _{\substack{|, J| \rightarrow 0 \\
J \subset I}} \sup \left\{|J|^{-1} \int_{J}\left|g-g_{J}\right|\right. \\
& \left.\quad+|J|^{-1} \int_{J}\left|\left(u_{\varepsilon}+\widetilde{v}_{\varepsilon}\right)-\left(u_{\varepsilon}+\widetilde{v}_{\varepsilon}\right)_{J}\right|\right\} \\
& \leqq 0+\left\|u_{\varepsilon}+\widetilde{v}_{\varepsilon}\right\|_{*} \\
& \leqq\left\|u_{\varepsilon}\right\|_{*}+\left\|\widetilde{v}_{\varepsilon}\right\|_{*} .
\end{aligned}
$$

Since the conjugation operator is a bounded map from $L^{\infty}$ into $B M O$, it follows that $\left\|u_{\varepsilon}\right\|_{*}+\left\|\widetilde{v}_{\varepsilon}\right\|_{*} \leqq K\left(2\left\|u_{\varepsilon}\right\|_{\infty}+\left\|v_{\varepsilon}\right\|_{\infty}\right)$. So, $\lim \sup _{|J| \rightarrow 0, J \subset I} 1 /|I|$ $\int_{I}\left|f-f_{I}\right| \leqq 2 K \cdot \varepsilon$, for some absolute constant $K$ and arbitrary $\varepsilon$. This means that $f$ is in $V M O(I)$, and (1) is true. The equivalence of properties (1) and (2) and that (2) implies (3) were established by elementary methods in [19]. Property (4) is an easy consequence of property (1).

Corollary 2. Let $w=|h|^{2}$ be in $W$. Then for every $x$ in $\boldsymbol{R}$, $\lim _{y \rightarrow 0}|(\bar{h} / h)(x+i y)|=1$ (by $(\bar{h} / h)(z)$ we mean the Poisson extension of $\bar{h} / h$ into the upper half-plane) and this convergence is uniform on bounded subsets of $\boldsymbol{R}$.

Proof. First of all, if $k$ is a bounded function on $\boldsymbol{R}$, it may be extended harmonically into the upper half-plane by $k(x+i y)=$ $\left(P_{y} * k\right)(x)$ where $P_{y}(t)=y / \pi\left(t^{2}+y^{2}\right)$. If $f$ and $g$ are bounded functions on the line and $f$ is continuous, then on every finite interval $J$,

$$
\lim _{y \rightarrow 0^{+}} \sup _{x \in J}|f(x+i y) \cdot g(x+i y)-(f g)(x+i y)|=0 .
$$

A proof of thit can be found in [18]. Let $\varepsilon>0$ be given and $J$ be fixed. Then we can write $\bar{h} / h=[(x-i) /(x+i)] e^{-2 i T x} b(x) e^{i(v(x)-\tilde{u}(x))}$ as in the first paragraph of the proof of Theorem 1. Now write $\bar{h} / h=f g$ where $g=e^{i(v-\tilde{u})}$ and $f=[(x-i) /(x+i)] / e^{-2 i T x} b(x)$. Then $f$ is continuous on $\boldsymbol{R}$, so by the remark at the beginning of this paragraph, it will suffice to show that $\lim _{y \rightarrow 0^{+}} \sup _{x \in R}|1-| g(x+i y)||<10 \varepsilon$ : this implies the inequality

$$
1-10 \varepsilon \leqq \lim _{y \rightarrow 0^{+}} \inf _{x \in J}\left|\frac{\bar{h}}{h}(x+i y)\right| \leqq \lim _{y \rightarrow 0^{+}} \sup _{x \in J}\left|\frac{\bar{h}}{h}(x+i y)\right| \leqq 1+10 \varepsilon
$$

for arbitrary $\varepsilon$. To get the desired inequality for $g$, write $g(x)=$ $k(x) e^{u(x)+i v(x)}$ where $k=e^{-u-i \tilde{u}}$, which is in $H^{\infty}$. Then $e^{-\varepsilon} \leqq|k(x)| \leqq e^{\varepsilon}$, 
and $\left|1-e^{u(x)+i v(x)}\right| \leqq 4 \varepsilon$, so

$$
\begin{aligned}
|k(x+i y)-g(x+i y)| & =\left|\int_{-\infty}^{\infty} k(t)\left\{1-e^{u(t)+i v(t)}\right\} P_{y}(x-t) d t\right| \\
& \leqq e^{\varepsilon} 4 \varepsilon .
\end{aligned}
$$

The desired inequality now follows easily for $\varepsilon<1 / 2$.

4. The class $W_{1}$. Let $W_{1}$ denote the class of integrable weights $w$ which can be expressed in the form $w=|B|^{2} e^{u+\tilde{v}}$ where $B$ is an entire function of exponential type which is square summable on $\boldsymbol{R}$, and where $u$ and $v$ are real bounded uniformly continuous functions. In this section, we show that $W_{1}$ is a subset of $W$, and a necessary and sufficient condition is given for a weight $w$ in $W$ to belong to $W_{1}$.

To see that $W_{1}$ is a subset of $W$ let $w=|h|^{2}$ be of the form $w=|B|^{2} e^{u+\tilde{v}}$ where $B, u$, and $v$ are as above. We can assume without loss of generality that $B$ has no zeros in the upper half-plane, so there exists a number $T$ such that $e^{i T x} B$ is an outer function in $H^{2}$. This implies that $\bar{h} / h=e^{-2 i T x}\left(B^{*} / B\right) \cdot e^{i(v-\tilde{u})}$; since $B^{*} / B$ essentially is a Blaschke product for the upper half-plane, it will suffice, by Lemma 1, to show that $e^{i(v-\tilde{u})}$ belongs to $H^{\infty}+B U C$. But $e^{i(v-\tilde{u})}=$ $e^{i v+u} \cdot e^{-u-i \tilde{u}}$. The first factor is in $B U C$ since $u$ and $v$ are, and the second factor is in $H^{\infty}$, so the desired result follows.

THEOREM 2. Let $w$ be in $W$, and $w=|h|^{2}$ where $h$ is outer in $H^{2}$. Then the following are equivalent:

(1) $w$ belongs to $W_{1}$.

(2) $\bar{h} / h$ can be factored as an inner function times a function which is invertible in $H^{\infty}+B U C$.

Before proving Theorem 2, it should be remarked that it is unknown whether or not condition (2) holds for all $w$ in $W$. It will be shown in Lemma 6 that (2) is "almost" true for every weight $w$ in $W$. This will be used to get a representation of the sort that defines the class $W_{1}$, except that the entire function $B$ will be of finite order. Next, two lemmas are given. A proof of Lemma 2 can be found in [6]. An argument from [18] is used in proof of Lemma 3.

LEMMA 2. Let $b$ be an inner function. Then $b$ is invertible in $H^{\infty}+B U C$ if and only if $b$ is of the form $b(x)=e^{i \sigma x} \cdot b_{0}(x)$ where $\sigma$ is a nonnegative number and $b_{0}$ is a Blaschke product whose zero sequence $\left(z_{n}\right)$ satisfies the inequality 


$$
\sup _{-\infty<x<\infty} \sum \frac{\operatorname{Im} z_{n}}{\left|x-z_{n}\right|^{2}}<\infty
$$

LEMMA 3. Let $\phi$ be a unimodular function in $H^{\infty}+B U C$. Then the following are equivalent:

(1) $\phi$ is invertible in $H^{\infty}+B U C$.

(2) $\operatorname{dist}\left(\bar{\phi}, H^{\infty}+B U C\right)<1$.

(3) $\phi=e^{-i T x} b(x) e^{i(u+\tilde{v})}$ where $T$ is a real number, $u$ and $v$ are real in BUC, and $b$ is a Blaschke product whose zero sequence $\left(z_{n}\right)$ satisfies $\sup _{-\infty<x<\infty} \sum \operatorname{Im} z_{n} /\left|x-z_{n}\right|^{2}<\infty$.

Proof. That (1) implies (2) is obvious. Suppose now that $\operatorname{dist}\left(\bar{\phi}, H^{\infty}+B U C\right)<1$. Then for some positive $T$, there is a $\psi$ in $H^{\infty}$ such that $\left\|e^{i T x} \bar{\phi}-\psi\right\|_{\infty}<1$, so $\left\|1-\phi e^{-i T x} \psi\right\|_{\infty}<1$. Now $\phi e^{-i T x} \psi$ is in $H^{\infty}+B U C$, so, by the last inequality, it must have a logarithm in $H^{\infty}+B U C$. Hence, we can write $\phi e^{-i T x} \psi=e^{f+i \tilde{f}+r+i s}$ where $f+i \tilde{f}$ is in $H^{\infty}$, and $r+i s$ belongs to BUC. Factoring $\psi$ as a product of its inner and outer parts, we get

$$
\psi=b e^{\log |\psi|+i \log |\psi| \sim},
$$

where $b$ is inner. Since $\left|\phi e^{-i T x}\right|=1$ a.e., we must have $f+r=$ $\log |\psi|$ a.e., so

$$
\dot{\phi}=e^{i T x} \bar{b} e^{i(\tilde{f}-\log |\psi| \sim)+i s}=e^{i T x} \bar{b} e^{i(s-\tilde{r})} .
$$

Now, $e^{i(\tilde{r}-s)}=e^{r+i \tilde{r}} \cdot e^{-r-i s}$, which belongs to $H^{\infty}+B U C$ since the first factor belongs to $H^{\infty}$ and the second to BUC. Thus $\dot{\phi}^{-1}=e^{-i T x} b e^{i(\tilde{r}-s)}$ and $\bar{b}=e^{-i T x} \phi e^{i(\widetilde{r}-s)}$ both belong to $H^{\infty}+B U C$. Thus conditions (1) and (2) are equivalent and imply that the function $\bar{\phi}$ satisfies (3). Therefore (1) implies that $\phi$ must satisfy (3) as well. It is clear that (3) implies (1), so the lemma is proved.

Proof of Theorem 2. Suppose that (2) in the statement of the theorem holds. Then by Lemma $3, \bar{h} / h=e^{-i T x} b e^{i(u+\tilde{v})}$ where $b$ is some inner function, and where $u$ and $v$ are in BUC. Now $e^{i u}$ can be uniformly approximated by entire functions of exponential type, so we can rewrite the last expression as $\bar{h} / h=e^{-i T x} \cdot F \cdot b \cdot e^{r+i(s+\tilde{v})}$ where $F$ is entire of exponential type, and where $r$ and $s$ are also in $B U C$, with $\|s\|_{\infty}<\pi / 2$. Then, we have

$$
|h|^{2} e^{-r-\tilde{s}+v}=e^{-i T x} h^{2} \cdot F \cdot b e^{-\tilde{s}+i s} \cdot e^{v+i \tilde{v}},
$$

and the proof of Theorem 1 can be adapted to show that

$$
|h|^{2}=\left(1+x^{2}\right)\left|B_{1}\right|^{2} e^{u_{1}+\tilde{v}_{1}}
$$


where $u_{1}$ and $v_{1}$ are real in $B U C$ and $B_{1}$ is an entire function of exponential type which is bounded on $R$. By letting $B_{2}=B_{1} /\left(z-z_{0}\right)$ for some zero $z_{0}$ of $B_{1},|h|^{2}$ can be written as

$$
|h|^{2}=\left(1+x^{2}\right)^{2}\left|B_{2}\right|^{2} e^{u_{2}+\tilde{v}_{2}}
$$

where $\left|B_{2}(x)\right|$ is now $O(1 / x)$ and hence square summable on $\boldsymbol{R}$. Now, the factor

$$
\left(1+x^{2}\right)^{2}=e^{2 \log \left(1+x^{2}\right)}=e^{-4[\arg (x+i)]^{\sim}}
$$

is of the form $e^{\tilde{t}}$ for $t$ in $B U C$. So putting this together yields $w=|h|^{2}=|B|^{2} e^{u+\tilde{v}}$ where $B=B_{2}, u=u_{2}$ and $v=v_{2}+t$, so $w$ be$\operatorname{logs}$ to $W_{1}$.

Suppose conversely that $w=|B|^{2} e^{u+\tilde{v}}$ as in (1) of Theorem 2. Then we can assume without loss of generality that $B$ has no zeros in the upper half-plane. This means that for some $T, e^{i T x} B$ is outer in $H^{2}$, so $\bar{h} / h=e^{-2 i T x}\left(B^{*} / B\right) e^{i(v-\tilde{u})}$. The factor $B^{*} / B$ is a Blaschke product, modulo a harmless exponential factor, so $\bar{h} / h$ is of the desired form by Lemma 3.

5. The class $W_{*}$. For a nonnegative weight function $w$ and real number $T$, define the number

$$
\rho^{*}(w, T)=\sup _{f, g}\left|\int_{-\infty}^{\infty} f(x) \bar{g}(x) w(x) d x\right|
$$

where $f$ and $g$ range, respectively, over the unit spheres of the subspaces $F_{0} \ominus\left(F_{0} \cap P_{T}\right)$ and $P_{T} \ominus\left(F_{0} \cap P_{T}\right)$ of $L^{2}(R, w d x)$. If $w$ is the spectral density of a stationary Gaussian process, then $\rho^{*}(w, T)$ measures something like the amount of dependence between "past" and "future" events conditional on the field generated by $F_{0} \cap P_{T}$ (see Dym and McKean [8]). Let $W_{*}$ denote the class of weight functions for which $\rho^{*}(w, T) \rightarrow 0$ as $T$ tends to infinity. At first glance, it may seem that $W$ is contained in $W_{*}$ however, this is not the case. An example is furnished at the end of this section. The weight functions in $W \cap W_{*}$ have a nice form which is given in the next theorem.

THeOREM 3. Let $w$ be an integrable weight function on $\boldsymbol{R}$. Then the following conditions are equivalent:

(1) $w$ belongs to $W \cap W_{*}$.

(2) $w$ belongs to $W$ and $\rho^{*}(w, T)<1$ for some $T$.

(3) $w$ belongs to $W_{*}$ and $\rho(w, T)<1$ for some $T$.

(4) $w(x)=\left(1+x^{2}\right)|B(x)|^{2} e^{u(x)+\tilde{v}(x)}$ where $u$ and $v$ are in real $B U C$, and where $B$ is an entire function of finite ex- 
ponential type which is square integrable on $\boldsymbol{R}$ and whose zero sequence $\left(z_{n}\right)$ satisfies

$$
\sup _{-\infty<x<\infty} \sum_{n=1}^{\infty} \frac{\left|\operatorname{Im} z_{n}\right|}{\left|x-z_{n}\right|^{2}}<\infty .
$$

Before proving Theorem 3, two lemmas will be stated and proved.

LEMMA 4. Let $A$ and $B$ be two closed subspaces of a Hilbert space $H$. Let $\rho(A, B)$ denote the cosine of the minimum angle between $A$ and $B$. If $H=A+B$ and $\rho(A, B)<1$, then $H=A^{\perp}+B^{\perp}$ and $\rho\left(A^{\perp}, B^{\perp}\right)=\rho(A, B)$.

Proof. Let $f$ be a unit vector in $A^{\perp}$. Let $\rho=\rho(A, B)<1$ and $\rho_{1}$ denote the length of the projection of $f$ on $B^{\perp}$. By assumption, $f=a+b$ where $a$ belongs to $A$ and $b$ belongs to $B$. Then the vectors $f, a$ and $b$ determine a right triangle whose hypotenuse has length $\|b\|$. It follows from elementary geometry that $\|b\|^{2} \leqq$ $1 /\left(1-\rho^{2}\right)$. Now, write $f=g+h$ with $g$ in $B$ and $h$ in $B^{\perp}$. It follows that $\|h\|=\rho_{1}$ and $\|g\|=\left(1-\rho_{1}^{2}\right)^{1 / 2}$. Thus, $1=\langle f, f\rangle=\langle b, f\rangle=$ $\langle b, g\rangle \leqq\|b\|\|g\| \leqq\left(1-\rho_{1}^{2}\right)^{1 / 2}\left(1-\rho^{2}\right)^{-1 / 2}$. This implies that $\rho_{1} \leqq \rho<1$. Hence, $\rho\left(A^{\perp}, B^{\perp}\right) \leqq \rho(A, B)<1$ so $A^{\perp}+B^{\perp}$ is a closed sum and $\left(A^{\perp}+B^{\perp}\right)^{\perp} \subseteq A \cap B=\{0\}$. This shows that $H=A^{\perp}+B^{\perp}$, and by symmetry, $\rho(A, B) \leqq \rho\left(A^{\perp}, B^{\perp}\right)$ so the lemma is proved.

LEMmA 5. Let $w=|h|^{2}$. Then $\rho^{*}(w, T)=\operatorname{dist}\left(e^{i T x} h / \bar{h}, H^{\infty}\right)$, so $w$ is in class $W_{*}$ if and only if $h / \bar{h}$ is in $H^{\infty}+B U C$.

Proof. Let $M_{T}=F_{0} \cap P_{T}$. It was noted in [7] that $M_{T}$ is the orthogonal complement in $L^{2}(R, w d x)$ of $N_{T}=\left(e^{i T x} / \bar{h}\right) H^{2}+(1 / h) \overline{H^{2}}$ and that the cosine of the minimum angle between the two summands of $N_{T}$ equals $\operatorname{dist}\left(e^{i T x} h / \bar{h}, H^{\infty}\right)$. Let $A$ denote the first summand of $N_{T}$ and $B$ the second, so $\rho(A, B)=\operatorname{dist}\left(e^{i T x} h / \bar{h}, H^{\infty}\right)$. Then a function $f$ belongs to $N_{T} \ominus A$ if and only if $f$ is orthogonal to $M_{T}$ and $\int_{-\infty}^{\infty} \bar{f}\left(e^{i T x} / \bar{h}\right) g \bar{h} h d x=0$ for every function $g$ in $H^{2}$. This last condition implies that $\bar{f} e^{i T x} h$ is in $H^{2}$, so $f$ is in $\left(e^{i T x} / \bar{h}\right) \overline{H^{2}}$ which equals $P_{T}$. Thus, $N_{T} \ominus A=P_{T} \ominus M_{T}$. A similar argument shows that $N_{T} \ominus B=F_{0} \ominus M_{T}$. An application of Lemma 4 to the Hilbert space $N_{T}$ now shows that

$$
\begin{aligned}
\rho(A, B) & =\rho\left(N_{T} \ominus A, N_{T} \ominus B\right) \\
& =\rho\left(P_{T} \ominus\left(F_{0} \cap P_{T}\right), F_{0} \ominus\left(F_{0} \cap P_{T}\right)\right)=\rho^{*}(w, T),
\end{aligned}
$$

provided that either $\rho(A, B)$ or $\rho^{*}(w, T)$ is less than 1 . This proves the lemma. 
Proof of Theorem 3. Let $w=|h|^{2}$. If any of the first three conditions of Theorem 3 are satisfied, then it follows from Lemmas 3 and 5 that the function $\bar{h} / h$ is invertible in $H^{\infty}+B U C$ and has the form $\bar{h} / h=e^{-i T x} b e^{i(u+\tilde{v)}}$ where $b$ is a Blaschke product for the upper half-plane whose zero sequence $\left(z_{n}\right)$ satisfies $\sup _{-\infty<x<\infty} \sum \operatorname{Im} z_{n} /$ $\left|x-z_{n}\right|^{2}<\infty$, and where $u$ and $v$ are real in BUC. Repeating the argument used to prove Theorem 1 shows that (4) holds since the nonreal zeros of the entire function $B$ are determined by the zero sequence of the Blaschke product $b$. Conversely, suppose that (4) holds. It can be assumed without loss of generality that $B$ has no zeros in the upper half-plane, so $\bar{h} / h=e^{i T x}[(x-i) /(x+i)] B^{*}(x) / B(x) e^{i(v-\tilde{u})}$ for some number $T$. It follows from Lemma 3 that $\bar{h} / h$ is invertible in $H^{\infty}+B U C$ and that the first three conditions of Theorem 3 must be satisfied, so the theorem is proved.

To see that $W$ is not contained in $W_{*}$, take an integrable weight of the form $w(x)=|B(x)|^{2}$ where $B$ is an entire function of finite exponential type which is square integrable on the real axis and has zeros with arbitrarily small (nonzero) imaginary part (see [8, p. 315]). Then, $\bar{h} / h=e^{-i T x} b(x)$ for some number $T$ and Blaschke product $b$ which is not invertible in $H^{\infty}+B U C$. It is then true then $\rho(w, T)=0$ but $w$ fails to belong to $W_{*}$. It is interesting to contrast this with the situation for weight functions on the unit circle. If $w$ is a nonnegative integrable function on the unit circle whose logarithm is also integrable, then, $w=|h|^{2}$ for some outer function in $H^{2}$ of the unit circle; the numbers $\rho(w, N)$ and $\rho^{*}(w, N)$ are defined in a corresponding way for each nonnegative integer $N$. Then $\rho(w, N)$ tends to zero if and only if the function $\bar{h} / h$ belongs to the algebra $H^{\infty}+C$ (where $C$ denotes the space of continuous functions on the unit circle), but $\rho^{*}(w, N)$ tends to zero whenever $\rho(w, N)$ does. This follows from a lemma due to Sarason (see [3, Theorem 2]) which states that if $u$ is a unimodular function on the unit circle and $\operatorname{dist}\left(u, H^{\infty}\right)=1$ but $\operatorname{dist}\left(u, H^{\infty}+C\right)<1$, then $\bar{u}$ belongs to $H^{\infty}[u]$.

6. Some necessary conditions for $w$ to be in $W$. We begin with a lemma which shows that condition (2) in Theorem 2 is almost satisfied by any $w$ in $W$.

Lemma 6. If $w=|h|^{2}$ is in $W$, and $G(x)$ is any unbounded increasing function on the positive reals, then it is possible to represent $\bar{h} / h$ in the following way:

$$
\bar{h} / h=b_{1} \bar{b}_{2} e^{i(s+\tilde{t})},
$$

where $s$ and $t$ are continuous functions on $\boldsymbol{R} \cup\{\infty\},\|s\|_{\infty}<\pi / 2$, and 
where $b_{1}$ and $b_{2}$ are inner functions with $\left|b_{2}^{\prime}(x)\right|=O(G(|x|))$ as $|x| \rightarrow \infty$.

Proof. The argument used here is a refinement of one given by Axler [1] to show that every function in $L^{\infty}$ is a quotient of a function in $H^{\infty}+C$ and a Blaschke product, where $C$ denotes the spece of continuous functions on $\boldsymbol{R} \cup\{\infty\}$. To begin with, $H^{\infty}+B U C$ can be generated by $H^{\infty}$ and the complex conjugate of any Blaschke product $b$ whose zero sequence $\left(z_{n}\right)$ satisfies $0<m \leqq \sum \operatorname{Im} z_{n}|| x-\left.z_{n}\right|^{2} \leqq$ $M<\infty$ for all real values of $x$ and some constants $m$ and $M$. For definiteness, let $\operatorname{Im} z_{n} \geqq 1$ for all $n$. Now, there are functions $h_{1}, h_{2}, \cdots$ in $H^{\infty}$ so that $\left\|\bar{h} / h-\bar{b}^{n} h_{n}\right\|_{\infty} \rightarrow 0$ as $n \rightarrow \infty$. Let $\gamma(r)$ be a function on the positive reals to be determined later, but with $0<\gamma(0)$, and with $\gamma(r)$ increasing and unbounded. For each integer $n$, there is an integer $M(n)$ such that for all real $x$

$$
\sum_{k=M(n)}^{\infty} \frac{\operatorname{Im} z_{k}}{\left|x-z_{k}\right|^{2}} \leqq \frac{\gamma(|x|)}{2^{n}}
$$

and

$$
\sum_{k=M(n)}^{\infty} \frac{\operatorname{Im} z_{k}}{\left|z_{k}\right|^{2}} \leqq 1 / 2^{n}
$$

Let

$$
b_{2}(z)=\prod_{n=1}^{\infty} \prod_{k=M(n)}^{\infty}\left(\frac{1-z / z_{k}}{1-z / \bar{z}_{k}}\right) .
$$

Then $b_{2}(z)$ converges and so is a Blaschke product. For each $n$, we can write $\bar{b}^{n} h_{n}=h_{n} \cdot a_{n} \bar{d}_{n} / b_{2}$ where $a_{n}$ is a Blaschke product, and $d_{n}$ is a finite Blaschke product, so $\bar{b}^{n} h_{n}=\psi_{n} / b_{2}$ where $\psi_{n}$ is in $H^{\infty}+C$. Since $\bar{b}^{n} h_{n}$ converges to $\bar{h} / h$ in $L^{\infty}, \psi_{n}$ must converge to some $\psi$ in $H^{\infty}+C$. Thus, $\bar{h} / h=\psi \bar{b}_{2}$, where the zero sequence $\left(\lambda_{n}\right)$ of $b_{2}$ satisfies

$$
\sum \frac{\operatorname{Im} \lambda_{n}}{\left|x-\lambda_{n}\right|^{2}} \leqq \gamma(|x|) \text { for all } x .
$$

Now, the Blaschke product $b_{2}(z)$ converges uniformly on compact subsets of the strip $|\operatorname{Im} z|<1$, so the formal differentiation

$$
b_{2}^{\prime}(x)=b_{2}(x) \sum_{n=1}^{\infty} \frac{2 i \operatorname{Im} \lambda_{n}}{\left|x-\lambda_{n}\right|^{2}}(-\infty<x<\infty)
$$

may be justified by Cauchy's integral formula for the derivative. Thus,

$$
\left|b_{2}^{\prime}(x)\right| \leqq \sum_{n=1}^{\infty} \frac{2\left|\operatorname{Im} \lambda_{n}\right|}{\left|x-\lambda_{n}\right|^{2}} \leqq 2 \gamma(|x|)
$$


so we get the desired estimate by letting $\gamma(|x|)=1+\sup \{0, G(|x|)\}$. Since $b_{2}$ is continuous across $R$ and $\bar{h} / h=\psi \bar{b}_{2}$, we must have, from Corollary 2, that $\psi$ is bounded away from zero in a neighborhood of $R$. So, by a theorem of Stegenga [21], we can write $\psi=$ $[(x+i) /(x-i)]^{n} b_{1} e^{2(s+\tilde{t})}$ where $b_{1}$ is an inner function, $n$ is an integer, and where $s$ and $t$ are $C$, with $\|s\|_{\infty}+\|t\|_{\infty}<\pi / 2$. Absorbing the factor $[(x+i) /(x-i)]^{n}$ into $\bar{b}_{2}$ does not change the asymptotic nature of $b_{2}^{\prime}(x)$, so the lemma is proved.

THeOREM 4. Let $w$ belong to $W$. Then $w$ can be written in the following form:

$$
w=\left(1+x^{2}\right) \cdot F \cdot e^{u+\tilde{v}},
$$

where $u$ and $v$ are continuous on $\boldsymbol{R} \cup\{\infty\}$, and where $F$ is an entire function of order at most 3 which is nonnegative, and bounded on $\boldsymbol{R}$.

Proof. From the previous lemma, we can let $\bar{h} / h=b_{1} \bar{b}_{2} e^{\lambda_{(s+\tilde{t})}}$ where $s$ and $t$ are in $C$, and $\left|b_{2}^{\prime}(x)\right|=O\left(x^{2}\right)$. Let $a(x)=\bar{b}_{2}(\sqrt[3]{x})$. Then $a$ is continuous on $\boldsymbol{R}$ and its modulus of continuity tends to zero at infinity, so by convolving with an appropriate member of Jackson's kernel $K_{\lambda}(x)=c_{\lambda}(\sin \lambda x / x)^{4}$, we get $a(x)=G(x)(1+\theta(x))$ where $G$ is entire of exponential type and $\theta$ is continuous on $R \cup\{\infty\}$ (see [20, p. 52]). Moreover, $\theta(x) \rightarrow 0$ as $x \rightarrow \infty$, and $\|\theta\|_{\infty}$ can be made arbitrarily small. Thus we write

$$
\bar{h} / h=b_{1}(x) G\left(x^{3}\right) e^{r \div i\left(s_{1}+\tilde{t}_{1}\right)}
$$

where $r, s_{1}$ and $t_{1}$ are in $C$, and $\left\|s_{1}\right\|_{\infty}<\pi / 2$. Now, by the same procedure as in the proof of Theorem 1 , the desired representation may be obtained.

The next theorem gives a representation for weights in $W$ in a closed form, but falls short of being a good generalization of the Helson-Sarason theorem.

Theorem 5. Let $w$ be a weight in $W$. Then there is a fixed $\delta>0$ such that $w$ can be represented in the form $w=\left(1+x^{2}\right) \mid B_{\mid}^{2} e^{u+\tilde{v}}$ where $B$ is an entire function of exponential type which is bounded and zero free in the upper half-plane, and where $\|u\|_{\infty}+\|v\|_{\infty}<\delta$, where $u$ and $v$ are functions in $C(w, B)$, the $C^{*}$-algebra generated by the inner functions which are invertible in $H^{\infty}\left[e^{-i x}, B^{*} / B\right]$. Furthermore, $C(w, B)=C(w)$ does not depend upon the representation and equals BUC if and only if $w$ also belongs to $W_{*}$. 
Before proving this theorem, the following technical lemma is needed.

Lemma 7. Let $A$ be a Douglas algebra and $A=H^{\infty}+C_{A}$. Then, if $f$ is in $A$, and $\varepsilon>0$, it is possible to write $f=k+g$ where $k$ is in $H^{\infty}, g$ belongs to $C_{A}$, and $\|g\|_{\infty}<(1+\varepsilon)\|f\|_{\infty}$.

Proof. If $f=k_{1}+g_{1}$ where $k_{1}$ is in $H^{\infty}$, and $g_{1}$ is in $C_{A}$, we have that $\operatorname{dist}\left(g_{1}, H^{\infty}\right) \leqq\|f\|_{\infty}$, so by part (iii) of the theorem in $\S 2$, there must be a function $k_{2}$ in $H^{\infty} \cap C_{A}$ with $\left\|g_{1}-k_{2}\right\|_{\infty} \leqq\|f\|_{\infty}(1+\varepsilon)$. Set $k=k_{1}+k_{2}$ and $g=g_{1}-k_{2}$ to get the desired result.

Proof of Theorem 5. Let $w$ belong to $W$. Then represent $w$ as in Theorem 1, i.e., $w=\left(1+x^{2}\right)|B|^{2} e^{s+\tilde{t}}$ where $\|s\|_{\infty}+\|t\|_{\infty}<\pi / 2$. Then for some number $T$, we can assume $e^{i T x} B$ is outer in $H^{\infty}$. Then $\bar{h} / h=e^{-2 i T x} \cdot[(x-i) /(x+i)] \cdot b \cdot e^{i(t-\tilde{s})}$ where $b$ is the Blaschke product associated with $B$. By Lemma $1, \bar{h} / h$ is in $H^{\infty}\left[e^{-i x}\right]$, so $e^{i(t-\tilde{s})}$ is in $H^{\infty}\left[\bar{b}, e^{-i x},(x+i) /(x-i)\right]=H^{\infty}\left[\bar{b}, e^{-i x}\right]$. Since $e^{s+i \tilde{s}}$ is in $H^{\infty}$, it follows that $e^{s+i t}=e^{s+i \tilde{s}} e^{i(t-\tilde{s)}}$ is in $H^{\infty}\left[\bar{b}, e^{-i x}\right]$. Since $\|t\|_{\infty}<\pi / 2, e^{s+i t}$ has a logarithm in $H^{\infty}\left[\bar{b}, e^{-i x}\right]$ by the functional calculus. By the theorem of Chang stated in $\S 2$, this logarithm has the form $f+g$ where $f$ is in $H^{\infty}$, and $g$ is in $C(w, B)$. By the last lemma, we may choose $f$ and $g$ so that $\|g\|_{\infty}<\pi / 2$. Thus, $e^{s+i t}=e^{(r+i \tilde{r})+(u+i v)}$ where $r+i \widetilde{r}$ is in $H^{\infty}$, and where $u$ and $v$ are real in $C(w, B)$ with $\|v\|_{\infty}<\pi / 2$. Now $r=s-u$, so $e^{i t}=e^{i(s-u)^{\sim}+i v}$, i.e., $e^{i(t-\widetilde{s})}=e^{i(v-\tilde{u})}$. This can happen only if $t-\widetilde{s}$ and $v-\widetilde{u}$ are equal modulo $2 \pi$. It will be shown next that $e^{s+\tilde{t}}=e^{u+\tilde{v}}$ up to a constant factor. To see this, note that you can write $0<|h|^{2} e^{-u-\tilde{v}}=e^{-2 i T x} h^{2} b e^{-u-\tilde{v}+i(v-\tilde{u})}$ a.e., and you can carry out the analytic continuation as in Theorem 1 , and get $|h|^{2}=S_{1} e^{u+\tilde{v}}$, where $S_{1}$ is entire, having the same zero set as $S=B B^{*}$ : The nonreal zeros all come from $b$, and the real zeros must be the same because $|S| /\left|S_{1}\right|$ and its reciprocal are locally in $L^{1 / 2}$ for $\delta<\pi / 2$, and the zeros have multiplicity 2 . This means that $S$ and $S_{1}$ can differ only by a factor of the form a. $\mathrm{e}^{c x}$, which, in view of the fact that $\left(\log S_{1}\right) /\left(1+x^{2}\right)$ and $(\log S) /\left(1+x^{2}\right)$ are integrable, must reduce to a constant. Hence, $w=\left(1+x^{2}\right)|B|^{2} e^{s+\tilde{t}}=$ $a\left(1+x^{2}\right)|B|^{2} e^{u+\tilde{v}}$ as desired. To see that $C(w, B)$ really only depends on $w$ for suitably small $\delta$ note that if

$$
\left(1+x^{2}\right)\left|B_{1}\right|^{2} e^{u_{1}+\tilde{v}_{1}}=\left(1+x^{2}\right)\left|B_{2}\right|^{2} e^{u_{2}+\tilde{v}_{2}}
$$

are two different representations of $w$, and, say, $\left\|u_{i}\right\|_{\infty}<1 / 4 \log 2$, and $\left\|v_{i}\right\|_{\infty}<\pi / 8$ (for $i=1,2$ ), then comparing the two resulting ex- 
pressions obtained for $\bar{h} / h$ shows that

$$
b_{1} \bar{b}_{2}=e^{i T x} e^{i\left[\left(v_{2}-v_{1}\right)+\left(u_{1}-u_{2}\right) \sim\right]}
$$

for some $T$, (where $b_{i}$ is the Blaschke product associated with $B_{i}$, $i=1,2)$. By the proof of Lemma 1 ,

$$
\operatorname{dist}\left(b_{1} \bar{b}_{2}, H^{\infty}\left[e^{-i x}\right]\right)<1 .
$$

Similarly,

$$
\operatorname{dist}\left(\bar{b}_{1} b_{2}, H^{\infty}\left[e^{-i x}\right]\right)<1 .
$$

This implies that $H^{\infty}\left[\bar{b}_{1}, e^{-i x}\right]=H^{\infty}\left[\bar{b}_{2}, e^{-i x}\right]$. The final claim follows from Lemma 3.

7. Discontinuous weights in $W$. Examples of unbounded weights in $W$ can be found in [12]. The following construction shows that a weight function in $W$ can have arbitrarily wild oscillatory discontinuities. Let $f_{n}$ be the function defined on $\boldsymbol{R}$ by $f_{n}(x)=1-|x|^{1 / n}$ for $|x|<1$, and $f_{n}(x)=0$ elsewhere. It is easy to check that $\left\|f_{n}\right\|_{*}<3 / n$. Furthermore, the $B M O$ norm $\|\cdot\|_{*}$ is invarient under linear change of variables. Now let $\left(r_{n}\right)$ be any sequence of real numbers which decreases strictly to zero, and let $\left(a_{n}\right)$ be chosen so that the intervals $\left[r_{n}-a_{n}, r_{n}+a_{n}\right]$ are disjoint. Define the function

$$
f(x)=\sum_{n=1}^{\infty} f_{2^{n}}\left[\left(x-r_{n}\right) / a_{n}\right] .
$$

Then $f$ belongs to $V M O$ and $w=e^{f} /\left(1+x^{2}\right)$ belongs to $W$ but has an oscillatory discontinuity at 0 which can be made arbitrarily wild by the choice of $\left(r_{n}\right)$.

ACKNOWLEDGMENT. I would like to express my gratitude to Donald Sarason for the advice and encouragement received in the preparation of this paper.

\section{REFERENCES}

1. S. Axler, Factorization of $L^{\infty}$ functions, Annals of Math., 106 (1977), 567-572.

2. R. P. Boas, Entire Functions, Academic Press, New York, 1954.

3. S.-Y. A. Chang, Structure of subalgebras between $L^{\infty}$ and $H^{\infty}$, Trans. Amer. Math. Soc., 277 (1977), 319-332.

4. S.-Y. Chang and D. E. Marshall, Some algebras of bounded analytic functions containing the disk algebra, Lecture Notes in Mathematics 604, Springer-Verlag, New York, 1977, 12-20.

5. P. L. Duren, Theory of $H^{p}$ Spaces, Academic Press, New York, 1970.

6. H. Dym, An extremal problem in the theory of Hardy functions, Israel J. Math., 18 (1974), 391-399. 
7. H. Dym, Trace formulas for a class of Toeplitz-like operators II, J. Functional Analysis, 28 (1978).

8. H. Dym and H. P. McKean, Gaussian Processes, Function Theory, and the Inverse Spectral Problem, Academic Press, New York, 1976.

9. C. Fefferman and E. M. Stein, $H^{p}$ spaces of several variables, Acta Math., 129 (1972), 137-193.

10. H. Helson and D. Sarason, Past and future, Math, Scand., 21 (1967), 5-16.

11. H. Helson and G. Szegö, A problem in prediction theory, Ann. Mat. Pure ed Appl., 51 (1960), 107-138.

12. I. A. Ibragimov, On the spectra of stationary Gaussian sequences which satisfy the strong mixing condition. I. Necessary conditions. Theor. Veroyatnost. i Primenen. 10 (1965), 95-116 (=Theor. Prob. Appl., 10 (1965), 85-106).

13. Conditions for the complete regularity of continuous time stationary processes, Sem. Math. V. A. Steklov Math. Inst. Leningrad, 12 (1971), 29-49.

14. P. Koosis, Moyennes quadratiques de transformée de Hilbert et fonctions de type exponentiel, C. R. Acad. Sci. Paris, 276 (1973), 1201-1204.

15. N. Levinson and H. P. McKean, Weighted trigonometrical approximation on the line with application to the germ field of a stationary Gaussian noise, Acta Math., 112 (1964), 99-143.

16. D. E. Marshall, Subalgebras of $L^{\infty}$ containing $H^{\infty}$, Acta Math., 137 (1976), 91-98.

17. D. Sarason, An addendum to "Past and Future", Math. Scand., 30 (1972), 62-64.

18. - Algebras of functions on the unit circle, Bull. Amer. Math. Soc., 79 (1973), 286-299.

19. - Functions of vanishing mean oscillation, Trans. Amer. Math. Soc., 207 (1975), 391-405.

20. H. S. Shapiro, Smoothing and Approximations of Functions, Van Nostrand Reinhold Co., New York, 1969.

Received May 22, 1979.

Fordham University

BRONX, NY 10458 



\section{PACIFIC JOURNAL OF MATHEMATICS}

\section{EDITORS}

DoNALD BABBITT (Managing Editor)

University of California

Los Angeles, CA 90024

Hugo Rossi

University of Utah

Salt Lake City, UT 84112

C. C. MOORE and ANDREw OGG

University of California

Berkeley, CA 94720
J. DugundJI

Department of Mathematics

University of Southern California

Los Angeles, CA 90007

R. FinN and J. Milgram

Stanford University

Stanford, CA 94305

ASSOCIATE EDITORS
R. ARENS
E. F. BECKENBACH
B. H. NeumanN
F. WOLF
K. YoshidA

\section{SUPPORTING INSTITUTIONS}

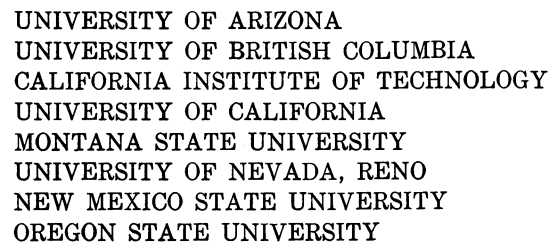

UNIVERSITY OF ARIZONA

UNIVERSITY OF BRITISH COLUMBIA

CALIFORNIA INSTITUTE OF TECHNOLOGY

UNIVERSITY OF CALIFORNIA

MONTANA STATE UNIVERSITY

UNIVERSITY OF NEVADA, RENO

NEW MEXICO STATE UNIVERSITY OREGON STATE UNIVERSITY

\author{
UNIVERSITY OF OREGON \\ UNIVERSITY OF SOUTHERN CALIFORNIA \\ STANFORD UNIVERSITY \\ UNIVERSITY OF HAWAII \\ UNIVERSITY OF TOKYO \\ UNIVERSITY OF UTAH \\ WASHINGTON STATE UNIVERSITY \\ UNIVERSITY OF WASHINGTON
}

The Supporting Institutions listed above contribute to the cost of publication of this Journal, but they are not owners or publishers and have no responsibility for its content or policies.

Mathematical papers intended for publication in the Pacific Journal of Mathematics should be in typed form or offset-reproduced, (not dittoed), double spaced with large margins. Please do not use built up fractions in the text of the manuscript. However, you may use them in the displayed equations. Underline Greek letters in red, German in green, and script in blue. The first paragraph or two must be capable of being used separately as a synopsis of the entire paper. Please propose a heading for the odd numbered pages of less than 35 characters. Manuscripts, in triplicate, may be sent to any one of the editors. Please classify according to the scheme of Math. Reviews, Index to Vol. 39. Supply name and address of author to whom proofs should be sent. All other communications should be addressed to the managing editor, or Elaine Barth, University of California, Los Angeles, California, 90024.

50 reprints to each author are provided free for each article, only if page charges have been substantially paid. Additional copies may be obtained at cost in multiples of 50 .

The Pacific Journal of Mathematics is issued monthly as of January 1966. Regular subscription rate: $\$ 102.00$ a year (6 Vols., 12 issues). Special rate: $\$ 51.00$ a year to individual members of supporting institutions.

Subscriptions, orders for numbers issued in the last three calendar years, and changes of address shoud be sent to Pacific Journal of Mathematics, P.O. Box 969, Carmel Valley, CA 93924, U.S.A. Old back numbers obtainable from Kraus Periodicals Co., Route 100, Millwood, NY 10546.

\footnotetext{
PUBLISHED BY PACIFIC JOURNAL OF MATHEMATICS, A NON-PROFIT CORPORATION

Printed at Kokusai Bunken Insatsusha (International Academic Printing Co., Ltd.). 8-8, 3-chome, Takadanobaba, Shinjuku-ku, Tokyo 160, Japan.
} 


\section{Pacific Journal of Mathematics}

\section{Vol. 96, No. 2 December, 1981}

Gerald A. Beer, A natural topology for upper semicontinuous functions and

a Baire category dual for convergence in measure $\ldots \ldots \ldots \ldots \ldots \ldots 251$

Georgia Benkart and J. Marshall Osborn, An investigation of real

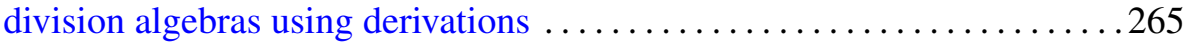

Donald Ian Cartwright and John R. McMullen, A structural criterion for the existence of infinite Sidon sets ........................ 301

Philip Hanlon, The fixed-point partition lattices $\ldots \ldots \ldots \ldots \ldots \ldots \ldots \ldots$

Eric Hayashi, The spectral density of a strongly mixing stationary Gaussian process

Chung-Wu Ho and Charles E. Morris, Jr., A graph-theoretic proof of

Sharkovsky's theorem on the periodic points of continuous functions . ...361

Sara Hurvitz, The automorphism groups of spaces and fibrations ....... 371

Atsushi Inoue, Schoichi Ota and Jun Tomiyama, Derivations of operator algebras into spaces of unbounded operators . .................. 389

Wolfgang B. Jurkat and Gary Sampson, On weak restricted estimates and endpoint problems for convolutions with oscillating kernels. I ........ 405

Georgios Koumoullis, Some topological properties of spaces of

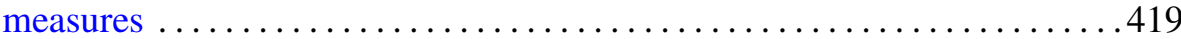

Wen Hsiung Lin, Algebraic Kahn-Priddy theorem ................. 435

Michael John McAsey, Invariant subspaces of nonselfadjoint crossed

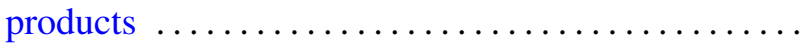

Justin Peters, Entropy of automorphisms on L.C.A. groups

Saburou Saitoh, A characterization of the adjoint $L$-kernel of Szegó type 